\title{
NOTE CRITIQUE SUR LA MALADIE DÉNOMMÉE
}

\author{
TICK PARALYSIS
}

\author{
Par P. PAVLOV
}

Parmi les maladies provoquées par les tiques, la « tick paralysis » présente un intérêt tout spécial au point de vue étiologique. Jusqu'à présent, cette maladie a été observée en Amérique du Nord, en Afrique du Sud, en Australie et en Europe dans la péninsule balkanique seule: Grèce, Yougoslavie et Bulgarie. Des auteurs russes l'ont également observée dernièrement en U.R.S.S., où elle est provoquée par la morsure d'Ornithodorus lahorensis. La « tick paralysis 》 a été décrite par Rastegaeva en 1940, Zelisteva en 1946 et Mamikonjan en 1946 également. Elle diffère de la maladie classique par ses symptômes cliniques, l'espèce et le nombre des tiques qui la provoquent, et par la durée de l'incubation. En voici un exposé critique.

Dans la littérature, on a jusqu'alors incriminé les femelles adultes d'Ixodes pilosus, I. holocyclus, Dermacentor andersoni et Hæmaphysalis punctata. Or, dans les cas décrits par Rastigaeva, Zelisteva et Mamikonjan, ce sont les Ornithodorus lahorensis au stade nymphal qui sont les agents de l'infection.

D'autre part, je tiens à souligner que la « tick paralysis » peut être provoquée par la morsure d'un seul spécimen des espèces mentionnées, s'il siège sur la tête ou l'épine dorsale, tandis que les Ornithodorus lahorensis doivent se fixer en grand nombre, ce qui fait penser à une intoxication et à une anémie, mais différentes de celles de la « tick paralysis » vraie.

La « tick paralysis » a une période d'incubation de 4-6 jours après la fixation de la tique femelle, tandis que la maladie étudiée expérimentalement sur le chien par Rastegaeva ne se manifeste que 21-23 jours après la fixation des tiques au stade larvaire, quand elles arrivent au $3^{e}$ stade nymphal. D'après le même auteur, si les Ornithodorus sont au $3^{\circ}$ stade nymphal ou à l'état adulte, la période d'incubation est de 23 jours chez le chien.

Pour Mamikonjan, la maladie se manifeste par une forte dépres-

Ann, de Parasitologie, T. XXII, Nos $5-6,1947$, p. $345-347$. 
sion qu'on n'observe pas dans la « tick paralysis » vraie. Zelisteva l'a également constatée, mais, dans mes observations personnelles, les animaux n'étaient déprimés que pendant quelques heures, 24 heures et non plus 48 heures, après l'arrachement des tiques de la tête et de l'échine, celles des, autres parties du corpö restant fixées, et sans aucun traitement.

Selon les auteurs mentionnés, si la maladie n'est provoquée que par des nymphes au $3^{\circ}$ stade nymphal, e'est qu'à ce stade seulement les tiques sont susceptibles d'intoxiquer; mais Mamikonjan écrit que la paralysie est constatée surtout chez les moutons attaqués par des tiques au stade nymphal. Dans ces conditions, la maladie pourrait donc être également provoquée par des tiques à un autre stade de développement, ce qui réfute l'opinion que j'ai émise en premier.

Il est vrai que Zelisteva constate, parmi les modifications anatomopathologiques, une diathèse hémorragique qui est en faveur d'une intoxication, mais la toxine en cause peut différer de celle de la « tick paralysis », où la diathèse hémorragique n'existe pas.

Pour Rastegaeva, la mort des animaux est en relation avec la quantité de toxine inoculée par les tiques au lieu de fixation. Mais, pour ma part, je ne pense pas que ce soit la quantité de toxine qui intervienne dans la manifestation de la maladie et la mort terminale, car la maladie devrait suivre son cours après l'arrachement des tiques ou de la tique fixées sur la tête, et conservation des tiques fixées sur le corps. A ce moment, en effet, les tiques du corps étant plus nombreuses inoculent une plus grande quantité de toxine. Or, on observe toujours la guérison en $\mathbf{2 4}$ heures, quand les tiques de la tête sont arrachées.

Dans ces conditions, je pense que la maladie décrite par les auteurs ci-dessus et provoquée par l'Ornithodorus lahorensis est une intoxication accompagnée d'anémie, différente de la «tick paralysis 》 vraie.

Au point de vue étiologique, la toxine responsable de la « tick paralysis », qui semble bien une intoxication, est différente des toxines déjà connues chez les autres arthropodes. En effet, la piqûre n'entraîne aucun symptôme local au lieu d'inoculation, ce qui est déjà observé dans le cas des Latrodectus; mais, dans ce cas, les symptômes cliniques qui suivent la piqûre sont tout à fait différents.

En conclusion, nous avons donc vu que la «tick paralysis" n'est pas déclanchée par l'inoculation en grande quantité d'une toxine éventuelle, mais exclusivement par la localisation céphalique et rachidienne d'une seule tique parfois, dont la suppression 
entraìne la guérison, même si des centaines de ces mêmes tiques demeurent fixées sur le corps. Aussi, cette toxine aura-t-elle un grand intérêt scientifique quand on l'aura découverte.

\section{BIBLIOGRAPHIE}

Brumpt (E.). - Précis de Parasitologie, 5e édition, Paris, Masson et Cie, 1936.

Mamikonjan (M. M.). - Les tiques Ornithodorus lahorensis et la paralysie du mouton provoquée par cette tique. $\mathrm{XXV}^{\text {e }}$ Plenum de la section vétérinaire de l'Académie d'Agriculture en U.R.S.S., p. 18, 1946.

Pavlov et Mrlowsky. - Deutsch. Tier. Woch., L, 1942, p. 539.

Rastegaeva (E. F.). - Observations expérimentales sur la paralysie à tiques chez le mouton. Deuxième conférence sur les problèmes parasitologiques. Acad. des Sciences de l'U.R.S.S. Section biologique, p. 29, 1940.

Zelisteva (L. M.). - La paralysie à tiques chez le mouton au Kasakstan. $X X V^{\bullet}$ Plenum de la section vétérinaire de l'Acad. d'Agriculture en U.R.S.S., p. 16, 1946.

La littérature sur la « tick paralysis » est mentionnée dans la publication de Pavlov et Miljowsky.

Institut central vétérinaire bactériologique de l'Etat, Sofia, Bulgarie (Chef du service antiparasitaire: P. Pavlov). 